\title{
Outcomes of surgically treated infective endocarditis in a Western Australian population
}

\author{
Aditya Eranki ${ }^{* *} \mathbb{D}$, Ashley R. Wilson-Smith ${ }^{1,2}$, Umar Ali $^{3}$, Akshat Saxena $^{3}$ and Eric Slimani
}

\begin{abstract}
Background: Infective endocarditis is a disease that carries high morbidity and mortality. The primary endpoint of this study is to assess factors associated with in-hospital mortality in patients undergoing valvular surgery for infective endocarditis. The secondary endpoint of this study is to assess the incidence of post-operative stroke, renal failure, complete heart block and recurrence.

Methods: Between the years of 2015 to 2019, a total of 89 patients underwent surgery for infective endocarditis at Fiona Stanley Hospital, Western Australia. Data was collected from the Australia and New Zealand Cardiac Surgery Database from 2015 to 2019 as well as patients electronic medical record. A number of preoperative and perioperative factors were assessed in relation to patient mortality and morbidity. Univariate and multivariate logistical regression analysis was done to assess for the association between factors and in-hospital morbidity and mortality.

Results: A total of 89 patients underwent surgery for infective endocarditis from 2015 to 2019, affecting a total of 101 valves. The mean age of patients was 53.7 \pm 16.5 . A total of 79 patients had a positive blood culture pre-operatively, with Staphylococcus Aureus being the most frequently cultured organism (39\%). Fourteen patients (16\%) were deemed emergent and underwent surgery within $24 \mathrm{~h}$ of review. A total of five patients died within their hospital stay postoperatively. Variables significantly associated with mortality on univariate analysis were intravenous drug use, emergent surgery, perioperative dialysis, perioperative inotropes, cardiopulmonary bypass time and cross clamp time. Only CBP time was significantly associated with mortality on multivariate analysis. A total of 19 patients (21\%) required hemodialysis after surgery, 10 patients sustained a postoperative stroke (11\%), 11 patients developed a complete heart block post operatively (12\%) and endocarditis recurred in 10 patients (11\%).

Conclusion: Prolonged cardiopulmonary bypass times were significantly associated with mortality. This study is novel to report a lower mortality rate than previously quoted in the literature. We also report our findings of organisms, preoperative embolic phenomena and surgery in a Western Australian population. We recommend that all patients with endocarditis are discussed in multidisciplinary forum.
\end{abstract}

Keywords: Infective endocarditis, Valvular heart surgery, Cardiac Surgery, Mortality, Outcomes

\section{Background}

Infective endocarditis (IE) is associated with a high morbidity and mortality, with the mortality rate in studies ranging between 6 and 25\%[1-5]. Patients can suffer

*Correspondence: adit.eranki@gmail.com

${ }^{1}$ Department of Cardiothoracic Surgery, John Hunter Hospital, Newcastle, Australia

Full list of author information is available at the end of the article from complications such as embolization, stroke, heart failure and cardiogenic shock, disseminated infection, abscess formation and arrythmias, including complete heart block. Factors associated with these complications have also been assessed in literature, although to a lesser extent [2-14]. Emergent surgery is often required, ranging between 25 and $50 \%$ of these cases $[4,7]$. The indications for early or emergent surgery in IE has been outlined recently by the European Society of Cardiology, 
with cardiogenic shock, high-risk vegetations and locally uncontrolled infection being the primary factors considered [2]. Grey areas exist with regards to timing of surgery in the setting of a pre-operative embolic stroke [2]. The concept of a 'Heart Team' comprising of various specialties to create management plans for individual cases has been shown to decrease mortality in these cases [2]. In this report, we describe our experience of surgically treated endocarditis in a single Australian institution with a comprehensive overview of organisms, sites of embolization, operation performed and surgical outcomes. The primary outcome of this study is to assess factors associated with in-hospital mortality in patients with IE undergoing surgery. The secondary outcome of this study is to assess the factors associated with morbidity; namely post-operative stroke, renal failure and dialysis, complete heart block and recurrence.

\section{Methods}

Data was retrospectively collected from the Australian and New Zealand Cardiac Surgery database (ANZSCTS). Between the years of 2015 to 2019, a total of 89 patients underwent surgery for IE at Fiona Stanley Hospital. Cases of IE related to cardiovascular devices were excluded. Furthermore, cases of infective endocarditis were retrospectively confirmed using the modified Dukes Criteria and patients that did not meet these criteria were excluded [15]. A total of 9 patients were excluded. Preoperative and operative factors were identified and recorded through a combination of the ANZSCTS database and patient electronic medical record (EMR). These factors were selected as they are assumed to be associated with an increased rate of morbidity and mortality post-surgery. The definitions of these factors are in accordance with those set by the ANZSCTS database. The valvular procedure was documented from a combination of the ANZSCTS database and the patient electronic medical record. This includes whether the patient had an aortic procedure, aortic valve, mitral valve, tricuspid valve or pulmonary valve procedure. The number of valves affected was documented, as well as whether the index operation was a valvular replacement or repair. In valve replacements, the prosthesis type was recorded (mechanical or bioprosthetic). In patients who underwent valvular repair, details of the repair were recorded.

\section{Outcomes}

The primary endpoint was defined as 30-day mortality. The second endpoint was the incidence of, and factors associated with post-operative sequelae: cerebrovascular accident (CVA), post-operative hemodialysis, new complete heart block (CHB) and recurrence. CVA postoperatively was defined as the occurrence of a stroke or new central neurologic deficit (persisting $>72 \mathrm{~h}$ ) postoperatively. Recurrence was defined as a new diagnosis of endocarditis after the patient's index operation for endocarditis.

\section{Statistical analysis}

Descriptive statistics, including number of events, mean and standard deviation, were calculated for each preoperative and operative variable. Univariate analysis was conducted to identify variables significantly associated with either in-hospital mortality or morbidity. Categorical variables were assessed using the chi-squared $\left(\mathrm{x}^{2}\right)$ test to ascertain odds ratios. Fishers exact test was conducted when more than $20 \%$ of cells on the contingency table had an expected frequency less than five. Continuous variables were first assessed for normality and then with the independent $T$ test to assess for equality of means. Variables that were not normally distributed were assessed with the Mann-Whitney $\mathrm{U}$ test. $P$ values less than 0.05 were deemed as significant. Preoperative and operative variables that reached significance were then further assessed with multivariate logistic regression analysis using a binary logistic regression model. Statistical analysis was done on IBMM ${ }^{\circledR}$ SPSS statistics version 25.

\section{Results \\ Demographic data}

A total of 89 patients underwent surgery for IE, affecting 101 valves. The mean age of patients was 53.7, with a minimum age of 16 and a maximum age of 83 . Most of the patients were male $(n=67)$. A large portion of patients $(n=28)$ had a history of IVDU. Seventeen patients had a history of Diabetes. Fifteen patients identified as ATSI.

\section{Preoperative patient factors}

A total of 15 patients $(17 \%)$ were in cardiogenic shock perioperatively. Eleven patients $(12 \%)$ required perioperative hemodialysis, 20 patients (22\%) were in perioperative respiratory failure and 17 patients (19\%) had perioperative inotrope requirements. Twenty-seven patients had vegetations greater than $20 \mathrm{~mm}$ on echocardiography, 32 patients had vegetations between 10 and $20 \mathrm{~mm}$ and 30 were less than $10 \mathrm{~mm}$. Most patients who underwent surgery had left-sided disease (82 patients) with 7 patients undergoing surgery for right-sided disease (see Table 1). A total of 79 patients had a positive blood culture preoperatively, with Staphylococcus Aureus being the most cultured organism (39\%). Ten patients (11\%) had culture negative endocarditis. These results are summarized in Table 2. Embolic phenomena were present in 39 patients (44\%). The most common site of embolization noted was 
Table 1 Preoperative and operative variables

\begin{tabular}{|c|c|c|}
\hline Preoperative variable & Preoperative variables & Operative variables \\
\hline Age & Preoperative cardiogenic shock & Cardiopulmonary bypass time \\
\hline \multirow[t]{2}{*}{53.7 years } & Yes 15 & $137 \mathrm{~min}$ \\
\hline & No 74 & \\
\hline Gender & Preoperative dialysis & Cross clamp time \\
\hline Male 67 & Yes 11 & $102 \min$ \\
\hline Female 22 & No 78 & \\
\hline IVDU history & Preoperative respiratory failure & Aortic procedure \\
\hline Yes 28 & Yes 20 & Yes 13 \\
\hline No 61 & No 69 & No 76 \\
\hline Location & Preoperative inotrope requirement & Double valve replacement \\
\hline Rural 33 & Yes 17 & Yes 8 \\
\hline Metropolitan 56 & No 72 & No: 81 \\
\hline ATSI & Urgency & Mitral valve repair \\
\hline Yes 15 & Elective 13 & Yes 15 \\
\hline \multirow[t]{2}{*}{ No 74} & Urgent 62 & No 28 (replacement) \\
\hline & Emergent 14 & \\
\hline \multicolumn{3}{|l|}{ Diabetes } \\
\hline \multicolumn{3}{|l|}{ Yes 17} \\
\hline \multicolumn{3}{|l|}{ No 72} \\
\hline \multicolumn{3}{|l|}{ BMl } \\
\hline \multicolumn{3}{|l|}{27.8} \\
\hline \multicolumn{3}{|l|}{ NYHA status } \\
\hline \multicolumn{3}{|l|}{ NYHA 152} \\
\hline \multicolumn{3}{|l|}{ NYHA 211} \\
\hline \multicolumn{3}{|l|}{ NYHA 313} \\
\hline \multicolumn{3}{|l|}{ NYHA 413} \\
\hline \multicolumn{3}{|l|}{ Positive blood culture } \\
\hline \multicolumn{3}{|l|}{ Yes 79} \\
\hline \multicolumn{3}{|l|}{ No 10} \\
\hline \multicolumn{3}{|l|}{ Organism } \\
\hline \multicolumn{3}{|l|}{ Staph A: 35} \\
\hline \multicolumn{3}{|l|}{ Other: 54} \\
\hline \multicolumn{3}{|l|}{ Vegetation size $(\mathrm{mm})$} \\
\hline \multicolumn{3}{|l|}{ 0-10: 30} \\
\hline \multicolumn{3}{|l|}{ 10-20: 32} \\
\hline \multicolumn{3}{|l|}{$>20: 27$} \\
\hline \multicolumn{3}{|c|}{ Native or prosthetic valve infection } \\
\hline Native valve 68 & & \\
\hline Prosthetic valve 21 & & \\
\hline Left sided disease & & \\
\hline Yes 82 & & \\
\hline No 7 & & \\
\hline Preoperative embolic ever & & \\
\hline Yes 39 & & \\
\hline No 50 & & \\
\hline Cerebral Emboli & & \\
\hline Yes 23 & & \\
\hline No 66 & & \\
\hline Preoperative creatnine & & \\
\hline
\end{tabular}


Table 1 (continued)

Preoperative variable

Preoperative variables

Operative variables

134 micromol/L

Table 2 Organisms isolated

\begin{tabular}{ll}
\hline Organism (N= 79) & Number (\%) \\
\hline Staphylococcus Aureus & $35(29 \%)$ \\
Enterococcus Faecalis & $18(20 \%)$ \\
Streptococcus Mitis & $8(9 \%)$ \\
Streptococcus Mutans & $3(3.3 \%)$ \\
Streptococcus Anginosus & $2(2.2 \%)$ \\
Streptococcus gordonii & $2(2.2 \%)$ \\
Escherichia Coli & $2(2.2 \%)$ \\
Streptococcus Agalactiae & $2(2.2 \%)$ \\
Streptococcus Parasanguinis & $1(1.1 \%)$ \\
Streptococcus Sanguinis & $1(1.1 \%)$ \\
Staphylococcus Hominins & $1(1.1 \%)$ \\
Capnocytophaga Canimorsus & $1(1.1 \%)$ \\
Streptococcus Pneumonia & $1(1.1 \%)$ \\
Staphylococcus Epidermitis & $1(1.1 \%)$ \\
Streptococcus Oralis & $1(1.1 \%)$ \\
Haemophilus Influenzae & $1(1.1 \%)$ \\
Neisseria Gonorrhoeae & $1(1.1 \%)$ \\
Aerococcus Urinae & $1(1.1 \%)$ \\
Culture negative & $10(11 \%)$ \\
\hline
\end{tabular}

Table 3 Sites of embolic phenomenon

\begin{tabular}{ll}
\hline Embolic phenomenon $\left(\mathbf{N}=\mathbf{3 9}^{\mathbf{a}}\right)$ & Number \\
\hline Lungs & 5 \\
Spleen & 2 \\
Spine & 4 \\
Joints (septic arthritis) & 2 \\
Brain & 23 \\
Skin & 6 \\
Vascular & 3 \\
Kidney & 1 \\
Other & 4 \\
\hline
\end{tabular}

a 10 patients had multiple sites of embolization

the brain, noted in 23 patients (26\%). Other common sites were skin ( 6 patients), lungs ( 5 patients) and spine (4 patients). Ten patients had multiple sites of embolization. These results are summarized in Table 3.

\section{Operative data}

Fourteen patients (16\%) were deemed emergent and underwent surgery within $24 \mathrm{~h}$ of diagnosis, whereas
Table 4 Valve and procedure

\begin{tabular}{|c|c|c|c|c|}
\hline $\begin{array}{l}\text { Procedure/ } \\
\text { prosthesis }\end{array}$ & $\begin{array}{l}\text { Aortic } \\
\text { valve } \\
(\mathrm{N}=52)\end{array}$ & $\begin{array}{l}\text { Mitral } \\
\text { valve } \\
(\mathrm{N}=43)\end{array}$ & $\begin{array}{l}\text { Tricuspid } \\
\text { valve } \\
(\mathrm{N}=9)\end{array}$ & $\begin{array}{l}\text { Pulmonary } \\
\text { valve } \\
(\mathrm{N}=1)\end{array}$ \\
\hline Mechanical & 23 & 18 & 1 & \\
\hline Bioprosthetic & 29 & 10 & & 1 \\
\hline Bentalls & 11 & & & \\
\hline Hemiarch & 1 & & & \\
\hline Annuloplasty & & 12 & 5 & \\
\hline Suture repair & & 5 & 3 & \\
\hline Neochords & & 8 & & \\
\hline Commisuroplasty & & 1 & & \\
\hline Other & $2^{a}$ & & & \\
\hline
\end{tabular}

the majority of patients (62) underwent urgent surgery (within $72 \mathrm{~h}$ ). The mean cardiopulmonary bypass time was $137 \mathrm{~min}$, and the mean cross clamp time was 102 min. The aortic valve was most affected, with 23 patients undergoing a mechanical aortic valve replacement (AVR) and 29 undergoing a tissue AVR. The mitral valve was also frequently involved, with 18 patients receiving a mechanical mitral valve replacement (MVR) and 10 receiving a tissue MVR. We opted to repair the mitral valve in 15 patients (35\%). One patient underwent a mechanical tricuspid valve replacement (TVR) and one patient underwent a bioprosthetic pulmonic valve replacement. Not uncommonly, infection involved the aortic root, with 11 patients undergoing a Bentalls procedure (12\%). Eight underwent double valve replacement, seven of which received an AVR and MVR and one patient receiving an AVR and pulmonic valve replacement (see Table 4).

\section{Primary endpoint: factors affecting in-hospital mortality}

Five patients died within 30 days of their index operation (6\%). Variables associated with mortality on univariate analysis were IVDU (OR 10, $P=0.032$ ), emergent surgery (OR 9.95, $P=0.026$ ), preoperative dialysis (OR 44, $P=0.001$ ), preoperative inotropes (OR 21.8, $P=0.004)$, CPBT with $250.8 \mathrm{~min}$ for non-survivors compared to $130.5 \mathrm{~min}$ for survivors $(P<0.001)$ and cross-clamp time (CCT) with 175 min for non-survivors compared to $97.8 \mathrm{~min}$ for survivors $(P=0.006)$. Multivariate analysis revealed that only CBP time was 
a significant predictor of operative mortality, with an odds ratio of 1.05 per minute of additional bypass time (95\% CI 1.001-1.101, $P=0.046$ ). These results are outlined in Table 5. A further subgroup analysis was performed on factors associated with prolonged CPBT, demonstrating that prosthetic valve involvement, Staphylococcus Aureus infection and aortic surgery were significantly associated with prolonged CPBT. Aortic surgery was significant on multivariate analysis (OR 27.8, 95\% CI 3.94-200). This is outlined in Table 6.

\section{Secondary endpoint: factors affecting postoperative} morbidity

Nineteen patients $(21 \%)$ required hemodialysis after surgery. Perioperative cardiogenic shock and perioperative dialysis were significantly associated with post-operative dialysis, with an odds ratio of 9.35 (95\% CI 1.47-58.8, $P=0.018)$ and 20 (95\% CI 2.24-167, $P=0.007)$ respectively. A total of 10 patients sustained a postoperative CVA (11\%). A number of factors were significantly associated with postoperative stroke, however, none of these reached significance on multivariate analysis.

Table 5 Outcome 1-univariate analysis of in hospital mortality

\begin{tabular}{|c|c|c|c|c|c|}
\hline Variable assessed & Death $(N=5)$ & Survivors $(\mathrm{N}=84)$ & Odds ratio & $P$ value & Adjusted OR \\
\hline Age & 52.4 & 53.8 & & $P=0.852$ & \\
\hline Gender (male) & 4 & 63 & & $P>0.900$ & \\
\hline IVDU & 4 & 24 & $10.0(1.0-94)$ & $P=0.032$ & \\
\hline Rural patient & 3 & 30 & & $P=0.355$ & \\
\hline ATSI & 2 & 13 & & $P=0.196$ & \\
\hline Hx of diabetes & 1 & 16 & & $P>0.900$ & \\
\hline BMI & 28.0 & 27.7 & & $P>0.900$ & \\
\hline NYHA 4 & 2 & 11 & & $P=0.153$ & \\
\hline Positive blood culture & 4 & 75 & & $P=0.457$ & \\
\hline Staph A & 3 & 32 & & $P=0.378$ & \\
\hline Vegetation > 20 mm & 3 & 24 & & $P=0.161$ & \\
\hline Prosthetic valve involved & 3 & 18 & & $P=0.083$ & \\
\hline Left sided endocarditis & 5 & 77 & & $P>0.900$ & \\
\hline Embolic phenomenon & 2 & 37 & & $P>0.900$ & \\
\hline Cerebral emboli & 1 & 22 & & $P>0.900$ & \\
\hline Preoperative creatinine & 118 & 135 & & $P=0.624$ & \\
\hline Emergent surgery & 3 & 11 & $9.95(1.49-66.4)$ & $P=0.026$ & \\
\hline Preoperative cardiogenic shock & 2 & 13 & & $P=0.196$ & \\
\hline Preoperative respiratory failure & 3 & 17 & & $P=0.073$ & \\
\hline Preoperative dialysis & 4 & 7 & $44.0(4.31-449)$ & $P=0.001$ & \\
\hline Preoperative inotropes & 4 & 13 & $21.8(2.26-211)$ & $P=0.004$ & \\
\hline Aortic procedure & 2 & 11 & & $P=0.153$ & \\
\hline Double valve replacement & 1 & 10 & & $P=0.602$ & \\
\hline Mitral valve repair & 0 & 14 & & $P=0.320$ & \\
\hline CPB time & 250.8 & 130.5 & $1.02(1.01-1.03)$ & $P<0.001$ & OR $1.05(1-1.1)$ \\
\hline Cross clamp time & 175 & 97.8 & $1.26(1.01-1.04)$ & $P=0.006$ & \\
\hline
\end{tabular}

Table 6 Prolonged CBPT

\begin{tabular}{|c|c|c|c|}
\hline Variable & Odds ratio & $P$ value & Adjusted OR \\
\hline Prosthetic valve endocarditis & $\begin{array}{l}N=8 \\
\text { OR } 3.19(\mathrm{Cl} 1.07-9.50)\end{array}$ & $P=0.032$ & \\
\hline Staph A & $\begin{array}{l}N=12 \\
3.50(1.22-10.1)\end{array}$ & $P=0.016$ & \\
\hline Aortic procedure & $\begin{array}{l}N=9 \\
\text { OR } 14.9(3.84-57.4)\end{array}$ & $P<0.001$ & $27.8(3.94-200)$ \\
\hline
\end{tabular}


Eleven patients developed a CHB post-operatively. All these patients required a pacemaker insertion. Factors associated with the development of $\mathrm{CHB}$ was crossclamp time (130 min vs $98 \min P=0.036)$ and whether the patient had an aortic procedure (OR 4.38, $P=0.05$ ). Infective endocarditis recurred in a total of 10 patients. Early recurrence (within a year of the index operation) occurred in four patients. None of these factors were significantly associated with recurrence. These results are outlined in Table 7.

\section{Discussion}

Surgical treatment for IE is associated with a high mortality rate, quoted between 6 and 25\% [3, 4, 7-12]. Risk factors associated with mortality include older age, emergent surgery, septic shock, congestive heart failure, cardiogenic shock, high risk organisms, prosthetic valve infection and stroke [1, 3-12, 16]. The European
Society of Cardiology (ESC) provide guidelines for the management of IE [2]. The guidelines advocate for early surgery in patients with heart failure, uncontrolled infection and high-risk lesions to prevent embolization [2]. Of all factors, congestive cardiac failure is the most consistent predictor of mortality [17, 18]. These studies advocate for early surgery in patients presenting in heart failure [17-19]. Early surgery for high-risk lesions is also supported by literature [20-22]. Of these, a randomized control trial by Kang et al. [21] demonstrated that early surgery in patients with large leftsided lesions $(>10 \mathrm{~mm}$ ) significantly reduced morbidity and embolic events. The ESC guidelines provide a class 1 indication for early surgery in vegetations greater than $10 \mathrm{~mm}$ with ongoing embolic phenomena. Uncontrolled infection is a further indication for early surgery. This is supported by several retrospective cohort

Table 7 Outcome 2-univariate analysis of morbidity

\begin{tabular}{|c|c|c|c|c|}
\hline Variable & Postoperative stroke $(\mathrm{N}=10)$ & $\begin{array}{l}\text { Postoperative dialysis } \\
(\mathrm{N}=19)\end{array}$ & $\begin{array}{l}\text { Recurrence } \\
(\mathrm{N}=10)\end{array}$ & $\begin{array}{l}\text { Complete } \\
\text { heart block } \\
(\mathrm{N}=11)\end{array}$ \\
\hline NYHA 4 & & $\begin{array}{l}N=7 \\
\text { OR } 6.22(1.8-22) \\
P=0.005\end{array}$ & & \\
\hline Preoperative emboli & $\begin{array}{l}N=8 \\
\text { OR } 6.19(1.23-31) \\
P=0.019\end{array}$ & & & \\
\hline Cerebral emboli & $\begin{array}{l}N=6 \\
\text { OR } 5.47(1.38-22) \\
P=0.017\end{array}$ & & & \\
\hline Preoperative creatinine & $\begin{array}{l}207 \text { versus } 125 \\
P 0.036\end{array}$ & & & \\
\hline Preoperative cardiogenic shock & $\begin{array}{l}N=5 \\
\text { OR } 6.90(1.7-28) \\
P=0.011\end{array}$ & $\begin{array}{l}N=10 \\
O R 14.4(4.0-52) \\
P<0.001\end{array}$ & & \\
\hline Preoperative respiratory failure & $\begin{array}{l}N=5 \\
O R 4.26(1.1-16) \\
P=0.042\end{array}$ & $\begin{array}{l}N=10 \\
O R 6.67(2.2-20) \\
P=0.001\end{array}$ & & \\
\hline Preoperative ionotropes & $\begin{array}{l}N=5 \\
\text { OR } 5.58(1.4-22) \\
P=0.020\end{array}$ & $\begin{array}{l}N=11 \\
O R 14.7(4.3-51) \\
P<0.001\end{array}$ & & \\
\hline Preoperative dialysis & & $\begin{array}{l}N=9 \\
O R 30.6(5.8-162) \\
P<0.001\end{array}$ & & \\
\hline Emergent procedure & $\begin{array}{l}N=4 \\
O R 4.60(C l 1.1-19) \\
P=0.047\end{array}$ & $\begin{array}{l}N=8 \\
O R 7.76(2.3-27) \\
P=0.001\end{array}$ & & \\
\hline Cross clamp time (min) & & & & $\begin{array}{l}130 \text { versus } 98 \\
P=0.036\end{array}$ \\
\hline RBC transfusion & & $\begin{array}{l}N=17 \\
O R 7.16(1.5-33) \\
P=0.007\end{array}$ & & \\
\hline Aortic procedure & & & & $\begin{array}{l}N=4 \\
O R 4.38(1.1-18) \\
P=0.05\end{array}$ \\
\hline
\end{tabular}


studies, demonstrating that locally aggressive infection is associated with a higher mortality rate [10,23].

Of these, a retrospective study by Revilla et al. [10] demonstrated that persistent infection is an independent predictor of mortality, where patients who undergo urgent surgery with persistent infection are four-fold as likely to die as patients without persistent infection. At Fiona Stanley hospital, we adopted these guidelines to help with decision making regarding operative timing. In the current study, the in-hospital mortality rate was 5.6\% or 5 out of 89 patients. This finding is novel as it is at the lower end of the spectrum of mortality figures quoted by other studies $[3,4,6,9,10]$. Rivas de Oliveira assessed 88 surgical patients between 2005 and 2015 and reported an in-hospital mortality rate of $17 \%$ [3]. Dunne et al. [11] in a similar Western Australian population with IE reported a mortality rate then of $13 \%$. One major change reported amongst hospitals during the last decade is the establishment of a dedicated "heart team". This team comprises of Cardiac Surgeons, Cardiologists and Infectious Diseases physicians. A dedicated "heart team" was established at Fiona Stanley Hospital since its initiation in 2015. Studies have reported a decline in mortality as a result of a multidisciplinary team (MDT) approach to endocarditis [24, 25]. A retrospective study by Chirillo et al. [24] demonstrated that after the implementation of an MDT, in-hospital mortality reduced from 28 to $13 \%$, as well as surgical mortality from 47 to $13 \%$. Similarly, a retrospective study conducted by Botelho-Nevers et al. [25] identified that MDT approach to endocarditis yielded a significant decrease in 1 -year mortality, from 18.5 to $8.2 \%$. There was also a statistically significant increase in compliance to antimicrobial therapy. The 2015 ESC guidelines (class 2 evidence) recommend the timing of surgical intervention via the consensus of an MDT team [2]. Our practice at Fiona Stanley Hospital is to conduct weekly MDT meetings to discuss cases of endocarditis which has potentially contributed to the low mortality rate.

Our study identified that IVDU, emergent surgery, perioperative dialysis, perioperative inotropes, prolonged cardiopulmonary bypass (CPB) time and prolonged CCT were significantly associated with in-hospital mortality on univariate analysis. This finding is consistent with previous studies $[1,3,6,11,26]$. СРВ time was the only factor to be significantly associated with death on multivariate analysis, with a mean CBP time of 250.8 vs $130.5 \mathrm{~min}$ for non-survivors and survivors respectively. A further analysis demonstrated that prosthetic valve involvement, Staphylococcus Aureus infection and aortic surgery was significantly associated with prolonged CPBT with aortic surgery reaching significance on multivariate analysis. Prolonged CPB time is a reflection of operative complexity, predisposes patients to end organ dysfunction, coagulation disorders and is therefore understandably associated with mortality.

Embolic phenomena occurred 39 patients (43.8\%). The most common site of emboli was the brain $(22$ patients) followed by skin and lungs. Other studies have also quoted equally high rates of embolic events [10, 27]. Likewise, in these studies, the brain was the most common site of embolism [10, 27]. Pre-operative stroke is a highly relevant complication of IE due to the risk of hemorrhagic transformation and postoperative neurological deterioration. Guidelines provide class 2A evidence to delay surgery by a month in the presence of intracranial haemorrhage [2]. As a result, we adopted a low threshold to conduct a CT brain, explaining the higher rate of cerebral emboli compared to other sites in this study. Embolic phenomena and cerebral emboli were linked to the incidence of preoperative stroke on univariate analysis, however, was not associated with in-hospital mortality.

In terms of organisms, Staphylococcus Aureus was most cultured and present in 39\% of patients. This was followed by Enterococcus Faecalis and Streptococcus Mitis in 20\% and 9\% of patients respectively. Eleven percent of patients had culture negative IE. The prevalence of Staphylococcus Aureus is a feature in other studies also $[6,10]$. There has been a reported shift in the epidemiology of IE away from Streptococcus and HACEK (Haemophilus species, Aggregatibacter species, Cardiobacterium hominis, Eikenella corrodens and Kingella) organisms towards Staphylococcus Aureus [28, 29]. This was also evident in our study, with only 15 patients culturing Viridians Streptococci. There was one case of HACEK endocarditis. Staphylococcus Aureus has been linked to a higher mortality rate in surgically treated endocarditis [30, 31]. It is also linked to locally aggressive infection, higher rates of embolization and septic shock [30, 31]. Our study did not demonstrate a relationship between Staphylococcus Aureus and in-hospital mortality or postoperative complications, however, we did demonstrate that may be linked with prolonged $\mathrm{CPBT}$ and operative complexity. At our institution, we favour early surgery for patients with Staphylococcus Aureus endocarditis.

The majority of our patients received a valve replacement. This was especially the case with aortic valve endocarditis, where all patients received a valve replacement. We opted to repair the mitral valve in 14 cases (33\%). The rate of repair is consistent with that reported in literature [32]. Mitral valve repair is associated with lower in hospital mortality and morbidity in literature, however, this was not reported in our study [32]. Twelve patients had endocarditis of the aortic root with periannular abscess formation. In cases such as this, we opted to perform radical debridement of the annulus followed by replacement of the aortic valve and root. In our centre, we opted 
to use a valved graft conduit in a Bentalls procedure, however, some studies advocate for allografts as they demonstrate a lower rate of postoperative graft infection [33]. Aortic surgery in endocarditis is associated with a high morbidity and mortality [34]. In our study, it was not significantly associated with mortality, though it was associated with longer CPBT and postoperative CHB. Surgery for right-side endocarditis was uncommon and was performed in 10 patients (11\%). Only four patients had isolated tricuspid valve replacements. All other cases of right-side disease were performed in conjunction with left-side valve surgery. One patient underwent a pulmonic valve replacement. Surgery for pulmonic valve endocarditis is rare and is most commonly performed on prosthetic infections of pulmonic valve allografts (Ross procedure) or in conjunction with other valves [35]. It is unusual to be performed in isolation [35]. Studies report excellent short- and long-term outcomes despite being an uncommon pathology [35]. In our case, it was performed with concurrent AVR.

Complications after surgery for IE were not uncommon. Ten patients $(11 \%)$ had a postoperative stroke. Identifiable risk factors were cerebral emboli, pre-operative creatinine, perioperative cardiogenic shock, perioperative respiratory failure, perioperative ionotropic requirement and emergent procedure. Other studies have demonstrated a similar incidence of post-operative stroke $[10,11,27]$. Only one other study investigated risk factors associated with post-operative stroke [11]. Post-operative stroke is a debilitating issue, and some centers advocate for delaying surgery to minimize the risk of hemorrhagic transformation [22, 31]. Others demonstrate that the overall mortality benefit from early surgery outweighs this risk [36]. The practice at Fiona Stanley Hospital was to delay surgery by a month if feasible if there is a significant risk of hemorrhagic transformation. A total of 19 patients (21\%) required dialysis postoperatively. On multivariate analysis, cardiogenic shock and pre-operative dialysis were independently associated with the incidence of post-operative dialysis. Post-operative renal failure is linked to a critical perioperative state and is associated with an increased risk of mortality [6, 10,37, 38]. Conduction abnormalities are an early indication of an infectious process expanding to involve the membranous interventricular septum, often in cases with aortic valve endocarditis. A total of 11 patients (12\%) had complete heart block, all of whom received a pacemaker. The incidence of which is comparable to that published in other studies [6,39].

This is a retrospective observational study with inherent biases in data collection. A larger prospective study may enable us to explore more factors associated with morbidity and mortality. Our small patient numbers and the small number of in-hospital deaths have limited the use of multivariate analysis to evaluate risk factors for inhospital mortality. Fiona Stanley Hospital is a new institution, and data is available over a period of 4 years. As a result, long term morbidity and survival data was not explored by this study and therefore Kaplan-Meier survival analysis was not conducted. Long term follow-up of our patients would be beneficial to assess whether the low in-hospital mortality rate is also translates into long term survival.

\section{Conclusion}

This study reports the morbidity and in-hospital mortality of 89 patients undergoing valvular surgery for IE at a single institution. Prolonged CPBT is significantly associated with mortality. Our study is novel in its reporting of a low 30-day mortality rate and exemplifies the need for a multidisciplinary approach to the management of endocarditis.

\section{Abbreviations \\ IE: Infective endocarditis; EMR: Electronic medical record; CCT: Cross clamp time; CPBT: Cardiopulmonary bypass time; ANZSCTS: Australia and New Zea- land Cardiac Surgery; IVDU: Intravenous drug use; ATSI: Aboriginal and Torres Strait Islander; BMI: Body mass index; NYHA: New York Heart Association; RBC: Red blood cell; NRBC: Non red blood cell; CVA: Cerebrovascular accident; AVR: Aortic valve replacement; MVR: Mitral valve replacement; TVR: Tricuspid valve replacement; ESC: European Society of Cardiology; MDT: Multidisciplinary team.}

\section{Acknowledgements}

Not applicable.

\section{Authors' contributions}

The corresponding author AE was involved with data collection, analysis and writing of the paper, the authors AS, UA and KS were involved in the subsequent editorial process. All authors read and approved the final manuscript.

\section{Funding}

Not applicable.

Availability of data and materials

The datasets used and/or analysed during the current study are available from the corresponding author on reasonable request.

\section{Declarations}

Ethics approval and consent to participate

Ethics approval was granted from the Hospitals review board (Approval Number 33939).

Consent for publication

Not applicable. There was no personalised patient information.

Competing interests

The authors declare that they have no competing interests.

\section{Author details}

${ }^{1}$ Department of Cardiothoracic Surgery, John Hunter Hospital, Newcastle, Australia. ${ }^{2}$ The Collaborative Research Group (CORE), Sydney, Australia. ${ }^{3}$ Department of Cardiothoracic Surgery, Fiona Stanley Hospital, Perth, Australia. 
Received: 5 July 2021 Accepted: 14 November 2021

Published online: 07 December 2021

\section{References}

1. Varela L, Lopez-Menedez J, Redondo A, Ricardo Fajardo E, Miguelena J, Martin M, et al. Mortality risk prediction in infective endocarditis surgery: reliability analysis of specific scores. Eur J Cardiothorac Surg. 2008;53(5):1049-54.

2. Habib G, Lancellotti P, Antunes M, Bongiorni M, Casalta J, Del Zotti F, et al. 2015 ESC guidelines for the management of infective endocarditis: the task force for the management of infective endocarditis of the European Society of Cardiology (ESC) Endorsed by: European Association for Cardio-Thoracic Surgery (EACTS), the European Association of Nuclear Medicine (EANM). Eur Heart J. 2015;36(44):P3075-3128.

3. Rivas de Olivera J, Arrais dos Santos M, Arnoni R, Ramos A, Togna D, Ghorayeb S, et al. Mortality predictors in the surgical treatment of active infective endocarditis. Braz J Cardiovasc Surg. 2018;33(1):32-9.

4. Prendergast B, Tornos P. Surgery for infective endocarditis. Circulation. 2010;121:1141-52.

5. Wang A, et al. Contemporary clinical profile and outcome of prosthetic valve endocarditis. JAMA. 2007;297(12):1354-61.

6. Farag M, Borst T, Sabashnikov A, Zeriouh M, Schmack B, Arif R, et al. Surgery for infective endocarditis: outcomes and predictors of mortality in 360 consecutive patients. Med Sci Monit. 2017;23:3617-26.

7. Hassan A, Sogomonian R, Zaiem F, Vyas N, El Hunjul M, Jolly J, et al. Morbidity and mortality of infective endocarditis in a hospital system in New York City serving a diverse urban population. J Investig Med. 2016;64(6):1118-23.

8. Tomos P, Lung B, Permanyer-Miralda G, Baron G, Delahaye F, Gohlke-Barwolf $C$, et al. Infective endocarditis in Europe: lessons from the Euro heart survey. Heart. 2005;91(5):571-5.

9. Cresti A, Chiaverelli M, Scalese M, Nencioni C, Valentini S, Guerrini F, et al. Epidemiological and mortality trends in infective endocarditis, a 17-year population-based prospective study. Cardiovasc Diagn Ther. 2017;7(1):27-35.

10. Revilla A, Lopez J, Vilacosta I, Villacorta E, Rollan M, Echevarria J, et al. Clinical and prognostic profile of patients with infective endocarditis who need urgent surgery. Eur Heart J. 2007;28(1):65-71.

11. Dunne B, Marr T, Kim D, Edwards M, Merry C, Larbalestier R, et al. Infective endocarditis. Heart Lung Circ. 2014;23:P628-635.

12. Wang T, Wang M, Pemberton J. Risk scores predicting mortality in surgery of infective endocarditis: a meta-analysis. Heart Lung Circ. 2016;25(2):296-7.

13. Karavas A, Filsoufi F, Mihaljevic T, Aranki S, Cohn L, Byrne J. Risk factors and management of endocarditis after mitral valve repair. J Heart Valve Dis. 2002;11(5):660-4.

14. Legrand M, Pirracchio R, Rosa A, Petersen M, Van der Laan M, Fabiani J, et al. Incidence, risk factors and prediction of post-operative acute kidney injury following cardiac surgery for active infective endocarditis: an observational study. Crit Care. 2013;17(5):P220.

15. Li JS, Sexton DJ, Mick N, Nettles R, Fowler VG Jr, Ryan T, et al. Proposed modifications to the Duke criteria for the diagnosis of infective endocarditis. Clin Infect Dis. 2000;30(4):633-63.

16. Thuny F, Salvo G, Belliard O, Avierinos J, Pergola V, Rosenberg V, et al. Risk of embolism and death in infective endocarditis: prognostic value of echocardiography: a prospective multicenter study. Circulation. 2005;112:P69-75.

17. Netzer R, Altwegg S, Zollinger E, Tauber M, Carrel T, Seiler C. Infective endocarditis: determinants of long term outcome. Heart. 2002;88:P61-66.

18. Nadji G, Rusinaru D, Remadi J, Jeu A, Sorel C, Tribouillou C. Heart failure in left-sided native valve infective endocarditis: characteristics, prognosis, and results of surgical treatment. Eur J Heart Fail. 2009;11(7):P668-675.

19. Vikram H, Buencconsejo J, Hasbun R, Quagliarello V. Impact of valve surgery on 6-month mortality in adults with complicated, left-sided native valve endocarditis: a propensity analysis. JAMA. 2003;290(24):P3207-3214.

20. Mangoni E, Adinolfi L, Tripodi M, Andreana A, Gamberdella G, Ragone E, et al. Risk factors for "major" embolic events in hospitalized patients with infective endocarditis. Am Heart J. 2003;146(2):P311-316.
21. Kang D, Kim Y, Kim S, Sun B, Kim D, Yun S, et al. Early surgery versus conventional treatment for infective endocarditis. N Engl J Med. 2012;366:P2466-2473.

22. Garcia-Cabrera E, Fernandez-Hidalgo N, Almirante B, Ivanova-Georgieva $R$, Noureddine M, Plata A, et al. Neurological complications of infective endocarditis: risk factors, outcome, and impact of cardiac surgery: a multicenter observational study. Circulation. 2013;127(23):2272-84.

23. Anguera I, Miro J, Vilacosta I, Almirante B, Anguita M, Munoz P, et al. Aorto-cavitary fistulous tract formation in infective endocarditis: clinical and echocardiographic features of 76 cases and risk factors for mortality. Eur Heart J. 2005;26(3):P288-97.

24. Chirillo F, Scotton P, Rocco F, Rigoli R, Borsatto F, Pedrocco A, et al. Impact of a multidisciplinary management strategy on the outcome of patients with native valve infective endocarditis. Am J Cardiol. 2013;112(8):1171-6.

25. Botelho-Nevers E, Thuny F, Casalta J, Richet H, Gouriet F, Riberi A, et al. Dramatic reduction in infective endocarditis-related mortality with a management-based approach. Arch Intern Med. 2009;169(14):1290-8.

26. Dohmen P, Binner C, Mende M, Bakhtiary F, Etz C, Pfannmuller B, et al. Outcome of aortic valve replacement for active infective endocarditis in patients on chronic hemodialysis. Ann Thorac Surg. 2015;99(2):532-8.

27. Pang P, Kong Sin Y, Hee Lim C, Tan TE, Lim S, Chao V, et al. Surgical management of infective endocarditis: an analysis of early and late outcomes. Eur J Cardiothorac Surg. 2015;74:826-32.

28. Jamil M, Sultan I, Gleason T, Navid F, Fallert M, Suffoletto M, et al. Infective endocarditis: trends, surgical outcomes, and controversies. JThorac Dis. 2019;11(11):P4875-4885.

29. DeSimone D, Tleyjeh I, Correa D, Anavekar N, Lahr B, Sohail M, et al. Temporal trends in infective endocarditis epidemiology from 2007 to 2013 in Olmsted County. MN Am Heart J. 2015;170(4):P830-6.

30. Remadi J, Habib G, Nadji G, Brahim M, Thuny F, Casalta J, et al. Predictors of death and impact of surgery in Staphylococcus aureus infective endocarditis. Ann Thorac Surg. 2007;83:P1295-1302.

31. Han S, Sorabella R, Vasan S, Grbic M, Lambert D, Prasad R, et al. Influence of Staphylococcus aureus on outcomes after valvular surgery for infective endocarditis. J Cardiothorac Surg. 2017;12:57.

32. Feringa $\mathrm{HH}$, Shaw $L J$, Poldermans $\mathrm{D}$, Hoeks $\mathrm{S}$, van der Wall EE, Dion RA, Bax JJ. Mitral valve repair and replacement in endocarditis: a systematic review of literature. Ann Thorac Surg. 2007;83(2):564-70.

33. Ting M, Wang C, Chi N, Hsu R, Chen Y, Yo H. Outcome for surgical treatment of infective endocarditis with periannular abscess. J Formos Med Assoc. 2020;119(1):P113-124.

34. Kirali K, Sarikaya S, Ozen Y, Sacli H, Basaran E, Yerlikhan OA, et al. Surgery for aortic root abscess: a 15-year experience. Tex Heart Inst J. 2016:43(1):20-8.

35. Liekiene D, Bezuska L, Semeniene P, Cypiene R, Lebetkevicius V, Tarutis $V$, et al. Surgical treatment of infective endocarditis in pulmonary position-15 years single centre experience. Medicina (Kaunas). 2019. https:// doi.org/10.3390/medicina55090608.

36. Murai R, Funakoshi S, Kaji S, Kitai T, Kim K, Koyama T, et al. Outcomes of early surgery for infective endocarditis with moderate cerebral complications. J Thorac Cardiovasc Surg. 2017;153:831-40.

37. Sheikh A, Elhenawy A, Maganti M, Armstrong S, David T, Fiendel C. Outcomes of surgical intervention for isolated active mitral valve endocarditis. J Cardiothorac Surg. 2009;137(1):P110-116.

38. Elmasry A, Omran A, Elprince A, Elameen S, Mansy M, Mahlab A. Predictors of in-hospital mortality in surgically treated valvular infective endocarditis cases at National Heart Institute. Egypt J Egypt Soc Cardio-Thorac Surg. 2017;25(1):P35-44.

39. Jassal D. Surgical management of infective endocarditis: early predictors of short-term morbidity and mortality. Ann Thorac Surg. 2006;82(2):524-9.

\section{Publisher's Note}

Springer Nature remains neutral with regard to jurisdictional claims in published maps and institutional affiliations. 